\title{
A study on an origami-based structure for use as a sun umbrella
}

\author{
Maria Savchenko $^{1}$ (D) Vladimir Savchenko ${ }^{2} \cdot$ Aya Abe $^{1} \cdot$ Ichiro Hagiwara $^{1} \cdot$ Phuong Thao Thai $^{3}$
}

Received: 25 December 2019 / Accepted: 9 June 2020 / Published online: 24 June 2020

(c) Springer Nature Switzerland AG 2020

\begin{abstract}
Origami art has found numerous engineering applications. Many studies show that folding structures are the source of the desired system characteristics. In this article, we discuss the design of a beach umbrella based on the principles of origami and the study of its optical and mechanical properties. The main requirements for the designed model are that it should reduce the harmful effects of solar radiation on human health, should be compact for storage, and not collapse in the wind. To demonstrate the optical and mechanical properties of the designed model, the developed recursive ray tracing algorithm is used to simulate the propagation of light rays through the designed paper origami-based structure and the numerical simulations are performed using the commercial software ANSYS and LS-DYNA to study the behavior of the designed origami-based structure under lateral forces as well as its folding behavior. A combination of ray tracing approach and an optimization technique based on the genetic algorithm for modifying the developed model is also discussed. Simulation results are presented in the illustrations.
\end{abstract}

Keywords Origami design - Geometric optics · Ray tracing technique - Optimization · Finite element simulation · Kinematic modelling

Mathematics Subject Classification $68 \mathrm{U} 05 \cdot 68 \mathrm{U} 20$

\section{Introduction}

The sun is a source of ultraviolet (UV), which has a beneficial and at the same time very harmful effects on human health. UV light is electromagnetic (EM) radiation with wavelengths $\lambda$ in the range of $100-400 \mathrm{~nm}$. The UV radiation with the wavelength range from 200 to $400 \mathrm{~nm}$ reaches Earth's surface through the atmosphere and the ozone layer.

According to the World Health Organization, UV radiation causes between 50 and $90 \%$ of skin cancers, strongly affects the immune system, and has a damaging effect on the retina of the eyeballs [1]. Radiation protection, such as sunscreens, sunglasses, umbrellas, wide-brimmed hats, or clothing, is necessary for everybody to avoid excessive exposure during their daily life. Umbrellas and beach tents provide limited sun protection. Any clothing cannot give $100 \%$ skin protection from UV light. If light is seen through the fabric to the lumen, it means that it also passes UV radiation. UV light is sufficiently retained by dense fabrics of cotton, flax, hemp, and natural silk fabrics do not protect against solar radiation. Bright or shiny clothing reflects more UV radiation than light and bleached cotton fabrics. Polyester material blocks ultraviolet maximally. A wool knitted fabric with the optimized parameters such as fiber diameter, yarn linear density, yarn twist, cover factor setting can provide high UV protection to human body [2]. Human skin can be exposed to solar radiation under clothing. Many protective agents, such as creams and ointments, are not waterproof and require repeated use after intense sweating with a negative effect on the skin. In

\footnotetext{
$\triangle$ Maria Savchenko, savchenko_maria@yahoo.com; tz12015@meiji.ac.jp | ${ }^{1}$ Meiji Institute for Advanced Study of Mathematical Sciences, Meiji University, Tokyo, Japan. ${ }^{2}$ Faculty of Computer and Information Sciences, Hosei University, Tokyo, Japan. ${ }^{3}$ Department of Applied Mechanics, Hanoi University of Science and Technology, Hanoi, Viet Nam.
} 
the open air, sunscreens, wide-brimmed hats, and umbrellas can be served based on special materials and structures with high SPF (Sun Protection Factor). Nevertheless, the problem of adequate protection from solar radiation remains, and decreasing the light propagation in the media is still an open question. As regards the mechanical behavior of sun protection umbrellas, it should be noted that they break due to strong wind pressure along the ribs that are the thin pieces of metal that are attached to the fabric. Preventing breakage due to a broken mechanism as a result of a strong wind is also an important subject for study.

The presented article is devoted to the study of a screen for protection from sunlight, such as a beach umbrella, which will reduce the harmful effects of solar radiation on human health and be resistant to wind pressure.

\section{Preliminary theoretical discussion}

The challenge is to create a light-protective structure that reflects solar radiation and can be used in the manufacture of solar umbrellas and wide-brimmed hats. Due to the short wavelengths of UV light compared to the geometrical parameters of the desired structure, the problem can be solved by means of geometric optics [3].

In this case, the reflective properties of the flat section boundaries are described by the Fresnel formulas [4, 5].

The reflection $r$ and the transmission $t$ coefficients for plane waves at an interface are typically obtained using Fresnel formulas. It needs to be noticed, that in the absence of light absorption $r+t=1$ in accordance with the law of conservation of energy.

The reflection coefficient $r$ is represented by the Fresnel formula:

$r=\frac{1}{2}\left[\frac{\sin ^{2}\left(\varphi-\varphi^{\prime \prime}\right)}{\sin ^{2}\left(\varphi+\varphi^{\prime \prime}\right)}+\frac{\tan ^{2}\left(\varphi-\varphi^{\prime \prime}\right)}{\tan ^{2}\left(\varphi+\varphi^{\prime \prime}\right)}\right]$

where $\varphi$ (the angle between the incident ray and the normal at the interface) and $\varphi^{\prime \prime}$ (the angle between the normal at the interface and refracted ray) are the angles of incidence and refraction.

When the light falls normally on the interface between two media $(\varphi=0)$, the coefficients $r$ and $t$ can be obtained using the Fresnel formulas as follows:

$r=\frac{\left(n_{2-} n_{1}\right)^{2}}{\left(n_{2+} n_{1}\right)^{2}}$

$t=\frac{4 n_{1}^{2}}{\left(n_{2}+n_{1}\right)^{2}}$

where $n_{1}, n_{2}$ are the indices of refraction of two media.
The relationship between refraction indices and the angles $\varphi$ and $\varphi^{\prime \prime}$ is described by Snell's law and according to the law of reflection, the angle of incidence $|\varphi|$ is equal to the angle of reflection $\left|\varphi^{\prime}\right|$ (the angle between the reflected ray and the normal at the interface). From Eq. (2) it follows that an increase in the absolute value of the difference $\left(n_{2}-n_{1}\right)$ leads to increasing light reflection at the interface. For summer sun umbrellas and wide-brimmed hats, usually light materials are used, i.e. materials with a relatively small absorption coefficient. In this case, the transmission coefficient is $t \approx 1-r$. Therefore, the task is equivalent to the problem of obtaining the maximum reflection coefficient. It follows that in the solar radiation range the surface with the non-profiled geometry does not provide a significant value of the reflection coefficient $r$ within the interval [0.9-1]. An analysis of the Fresnel formulas (Eqs. 1, 2) shows that the reflection coefficient $r$ increases markedly with increasing the angle of incident $\varphi$. Such a correlation between parameters $r$ and $\varphi$ can be used to create structures with high reflectance.

The wedge-shaped structure is a potentially promising periodic structure. Its characteristic feature is the provision of reflection with a large angle of incidence of the wave at all reasonably permissible positions of the source. The wave is reflected from the profiled structure several times, but with much lower reflection coefficients in each case. The number of reflections and the angles of incidence on the flat faces of the structure depends on the angle $\gamma$ at the apex of a wedge [6].

We consider three values of the angle $\gamma$ for the profiled periodic 2D wedge-shaped structure that is schematically shown in Fig. 1:

- For $\gamma=90^{\circ}$, the number of reflections of the wave incident from different directions is always two. As a result, the gain in the total reflection coefficient is $2-4$ times;

- For $\gamma<90^{\circ}$, the number of reflections increases with increasing the angle $\varphi$ on the flat faces. The problem arises of finding the extremum of a function of several variables (the values of the angles $\gamma$ and $\varphi$, the dielec-

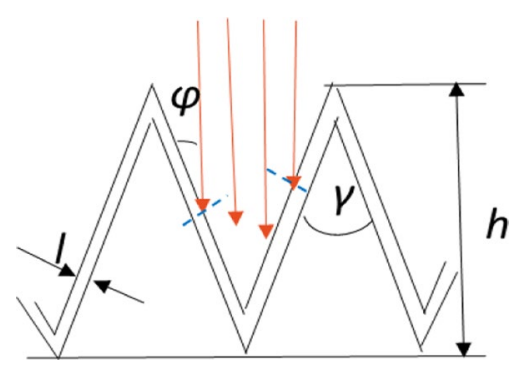

Fig. 1 The scheme of periodic profiled structure: the fragment of the 2D wedge-shaped structure (the red arrows indicate the incident light rays) 
tric constant of the medium, the thickness of the structure $l$, and the height of the wedges $h$ ) in the form of a reflection coefficient with constraints. Restrictions on the solution are acceptable ranges of structure parameters $(\gamma, l, h)$ and the values of the refractive index $n$ of the screen material. The minimum value of $h$ can be chosen from very small (a few wavelengths) to several $m m$ to simplify the technology in a practical implementation of the structure;

- For $\gamma>90^{\circ}$, the number of reflections turns out to be one or two in some cases. Compared with the smoothed structures, the angle $\varphi$ is increasing (equal to $\gamma / 2$ with normal incidence) that leads to decreasing transmission coefficient. The task is to look for the characteristics of the structure in a form of dependences of the reflection coefficient (or transmission) on the angle $\gamma$, the parameters $/$ and $h$ with restrictions taking into account a different number of reflections.

For the wedge-shaped structures, zones with a high concentration of the incident wave energy may appear at the apex of the wedge. This will increase the transmission coefficient in this area. To reduce the transmission coefficient, a smoother tip of the wedge is more suitable. It is possible to use the rough surfaces $(h \approx \lambda)$ with the regular surface profiles. Their use reduces the concentration of the energy of the incident wave at the apex of the wedge. In this case, probabilistic methods are required for calculations and modelling. Structures in the form of pyramids or cones tightly adjacent to each other and occupying the entire screen area can potentially be effective [7].

From the above discussion, it should be concluded:

- In the sunlight range the gain in the reflection coefficient for the periodic profiled structures can be 3-5 times or more in comparison with the non-profiled geometry (a smooth surface);

- The geometrical objects created on the basis of the origami principles can be considered as the profiled ones to increase the number of reflections with increasing the angle $\varphi$ on the flat faces.

Nowadays, origami-based engineering makes it possible to design structures with remarkable performance characteristics. In recent years, the origami approach has found application in many engineering areas, such as space structures, shelters, sandwich panels, meta-materials, healthcare, robotics, the origami-inspired EM structures [8-15]. A method to evaluate the mechanical performance of an origami-structure throughout the design process is presented in [16]. The method proposed in [17] can evaluate the kinematic indeterminacy and predict the folding behavior of symmetric frameworks.
Based on the foregoing, we can formulate the aim of the research as follows: to explore the possibility of applying an origami approach to create a sun-protection and windresistant structure.

The main objectives of the study are:

(a) To design an origami-based beach umbrella.

(b) To examine the properties of reflection/refraction of the designed origami-based structure.

(c) To simulate the mechanical and folding behavior of the structure under forces.

To solve the problems, the methods of geometric optics, ray tracing technique, finite element method (FEM), and finite element analysis (FEA) are used.

\section{Umbrella design}

There is a good deal of research on UV protection efficacy of the beach umbrellas and shade structures with UVblocking materials and UV-filter coating [18-22].

In our study, the designed origami-based beach umbrella is a flat-folded structure inspired by the simplicity of the Miura-ori unit-cell [23]. The folding/crease pattern is constructed as an eight-sided polygon with eight equal triangles. Each resulting triangle represents a unitcell with the facets, mountain creases, which are marked as red solid lines, and valley creases that are marked as dashed blue lines, as shown in Fig. 2a. Each unit-cell includes two inner vertices (the intersection points of the crease lines). This design satisfies the condition of Kawasaki's theorem [24] related with local flat-foldability that is $\beta_{1}+\beta_{3}=\beta_{2}+\beta_{4}=\pi$ for the single vertex (Fig. 2b), and Maekawa's theorem that states: the difference between the number of mountain creases and valley creases around a vertex is two. The 3D shape is obtained by folding the initial 2D pattern in a certain way. Cutting the pattern along the edge between two unit-cells is performed for the activation (the directions of the blue arrows show the direction that the forces are acting) of the developing process and forming a compact shape (Fig. 2c, d). For the formation of the final form of the origami-based umbrella, the end unit-cells are connected together, as shown in Fig. 2e. This basic umbrella model can be scaled or modified, for instance, by the design method presented in [25] for the $3 \mathrm{D}$ origami with an axisymmetric structure. The design parameters such as the distance between two inner vertices of the unit-cell and a number of unit-cells can be modified. For the numerical simulations, 3D polygonal models of origami and traditional umbrella (non-origami) were developed (Fig. 2f, g). The polygonal non-origami model includes 8 curved unit-cells (almost spherical and 


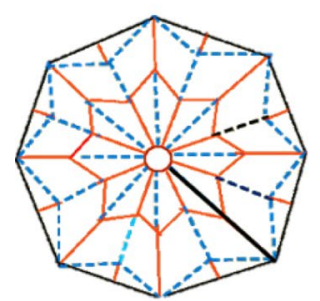

(a)

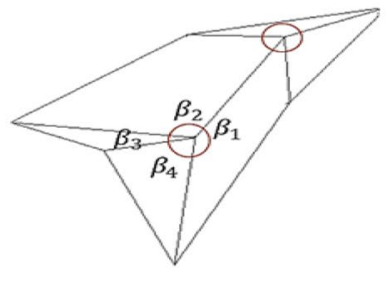

(b)

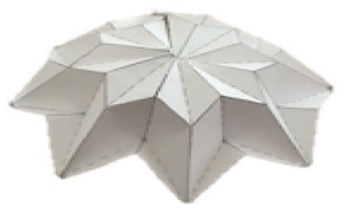

(e)

Fig. 2 The origami-based umbrella model: a the 2D crease pattern with the central hole (the cut line is indicated in bold black), $\mathbf{b}$ the 3D unit cell, $\mathbf{c}$ the folded paper model with intermediate folding forms, $\mathbf{d}$ the compact umbrella shape, e the paper umbrella

resembling traditional umbrella) with the smoothed surfaces without ribs or folding parts (the geometrical parameters: the diameter $\mathrm{D}$ and the height $\mathrm{H}$ shown below in Fig. 6a). The proposed origami-based model provides a sufficient number of folds to study its reflective/refractive properties (Sect. 4), the mechanical and folding behavior of the structure under the action of forces (Sect. 5).

\section{Ray tracing calculation and visualization of light intensities}

In geometric optics, an assumption is made that in a uniform media light travels in a straight-line path, which can be approximated by a ray, where the ray is a straight line perpendicular to the wavefronts. Ray tracing is extensively employed in designing optical instruments, analyzing radiation heat exchange through windows, in acoustics, etc. When the light wavelength is much less than the feature size of the medium, Maxwell's equations reduce to the eikonal equation, which is the basis of geometric optics [26] or the ray tracing method. In computer graphics, ray tracing is used for rendering the $3 \mathrm{D}$ objects by recursively following the path that the incident light takes. Traditionally, the 2D window (screen) is used for assigning a colour to each square of the view window.

Our goal is to calculate the light (wave) intensity values at the points of the 3D view "window". Volume visualization [27] as the most applicable visualization method is used to demonstrate the distribution of light rays in the areas below the structures under study. A cubic

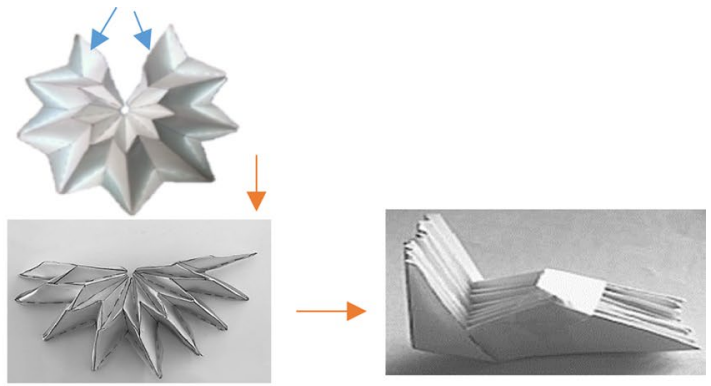

(c)

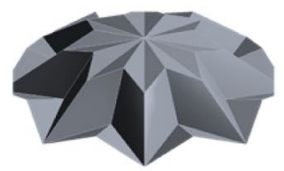

(f)

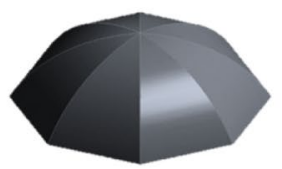

(g)

after the connection of the end unit-cells (with the suitable type of a lock), $\mathbf{f}$ the $3 \mathrm{D}$ origami polygonal model, $\mathbf{g}$ the 3D non-origami polygonal model

(voxel) volume represents the area of reception of each ray location. Following this concept, we define the 3D voxel volume with cells/voxels as identical subvolumes, where the actual data is accumulated. In our application, for convenience, we use an analog of a 3D chained list where we can store and modify radiation intensity (photon energy) or other data. In the developed Recursive Ray Tracing (RRT) algorithm, a set of refraction/ reflection events is treated as $n$-layers structure based on the Kubelka-Munk (K-M) method [28, 29] for modelling reflectance and transmittance. The RRT algorithm is implemented using the $\mathrm{C}++$ programming language and is designed to study the reduction of light propagation through the origami paper umbrellas.

In the current stage of the project, we consider only calculations of light intensity based on the detection of the intersections between rays and volume cells. However, the contribution of each individual ray to the resulting $3 \mathrm{D}$ vector field can allow one to calculate the electric field strength amplitude, phase, and polarization for each position on an arbitrary ray at a specified distance from the source. The reflected wave front is represented by a finite set of reflected rays depending on the intersected surface. The Oren-Nayar reflection model is used as a model for diffuse reflection [30]. For surface reflection, we use the surface roughness value equal to $0.1 \mathrm{~mm}$. Refraction is calculated in accordance with a quantity of the refraction indices by using Snell's law for a given pair of media. This process of reflection/ refractions continues iteratively. 


\subsection{Paper model}

Paper is a complex network of fibers, fiber fragments, and possibly fillers (Fig. 3). Multiple publications related to the paper structure and light propagation in it were presented, among them [31-33]. Despite the fact that the paper structure is extremely complex and it is hard to extract its features into a single parameter set, a crude approximation of the propagation of light in paper can be used, as it was well discussed in [34]. The K-M theory remains the most used in practice. Even though it has some disadvantages due to its explicit form, this theory is simple for using and gives acceptable prediction accuracy in many cases.

We suppose that the $\mathrm{K}-\mathrm{M}$ theory is sufficiently accurate to approximate light propagation in paper for the purpose of investigating light propagation in the origami-like objects. The optical material properties are condensed to two parameters: absorption $(K)$ and scattering $(S)$.

In our implementation of recursive ray tracing, a set of refraction/reflection events is treated as $n$-layer material obtained using the adding method [29]. The relationship between the coefficients $S, K$, and reflectances $R_{0}, R_{\infty}$ are determined as follows:

$$
\begin{aligned}
& R_{\infty}=1+\frac{K}{S}-\sqrt{\frac{K^{2}}{S^{2}}+2 \frac{K}{S}} \\
& R_{0}=\frac{e^{S W\left(\frac{1}{R_{\infty}}-R_{\infty}\right)}}{\frac{1}{R_{\infty}} e^{S W\left(\frac{1}{R_{\infty}}-R_{\infty}\right)}-R_{\infty}}
\end{aligned}
$$

where $R_{\infty}$ represents the reflectance of an opaque pile of homogenous paper and $R_{0}$ is the reflectance of a single sheet with black, totally absorbing, background. $W$ is the basis weight of the material. The light transmittance of the material is given as a function of light absorption, scattering coefficients, and $R_{\infty}$.

The theoretical relationship between the basis weight and light transmittance $T$ is as follows:

$$
T=\frac{\left(1-R_{\infty}^{2}\right) e^{1 / 2 S W\left(\frac{1}{R_{\infty}}-R_{\infty}\right)}}{1-R_{\infty}^{2} e^{-S W\left(\frac{1}{R_{\infty}}-R_{\infty}\right)}}
$$

The equations for $n$-layer material can be obtained using the adding method where the reflectance and transmittance of a two-layer material are defined when reflectance $R_{\mathrm{i}}$ and transmittance $T_{\mathrm{i}}$ at each layer are known.

The transmittance and reflectance for $n$-layers material are as follows:

$T_{1,2,3, \ldots, n}=\frac{T_{1} T_{2,3, \ldots, n}}{1-R_{1} R_{2,3, \ldots, n}}$

$R_{1,2,3, \ldots, n}=R_{1}+\frac{T_{1} R_{2,3, \ldots, n}}{1-R_{1} R_{2,3, \ldots, n}}$

The transmittance $T_{2,3, \ldots, n}$ and reflectance $R_{2,3, \ldots, n}$ can be computed recursively.

\subsection{Simulation of the light propagation}

A sheet of paper transmits visible light and probably other parts of the spectrum in the UV range as well. The light is scattered but it is being transmitted through the gaps between the paper fibers. The fibers are not absolutely opaque as can be seen in Fig. 3 .

The purpose of the simulation is to show the effectiveness of the origami-based structure to redirect light rays in comparison with the non-origami structure. When light is incident on a material surface, the light wave will either be reflected, transmitted, or absorbed. The propagation of light in the origami-based and non-origami umbrella models, by using the model of paper outlined above, is simulated by using the RRT algorithm. In the simulation, the 3D polygonal origami and non-origami models are embedded in a cube of size $2 \times 2 \times 2 \mathrm{~m}(128 \times 128 \times 128$ voxels). It is schematically shown in Fig. 4a. The geometrical parameters of the models are the diameter $D=1.7 \mathrm{~m}$; the height $\mathrm{H}=0.4 \mathrm{~m}$. The cube is divided into small subcubes with a side of $2 / 128 \mathrm{~m}$. The horizontal rows (from the top to bottom) of sub-cubes are called cube layers. Three
Fig. 3 Microscopic images of paper structure: the top view of the ordinary paper (left); the cross-section view of washi-paper ("Japanese paper") (middle); the top view of washi-paper (right)
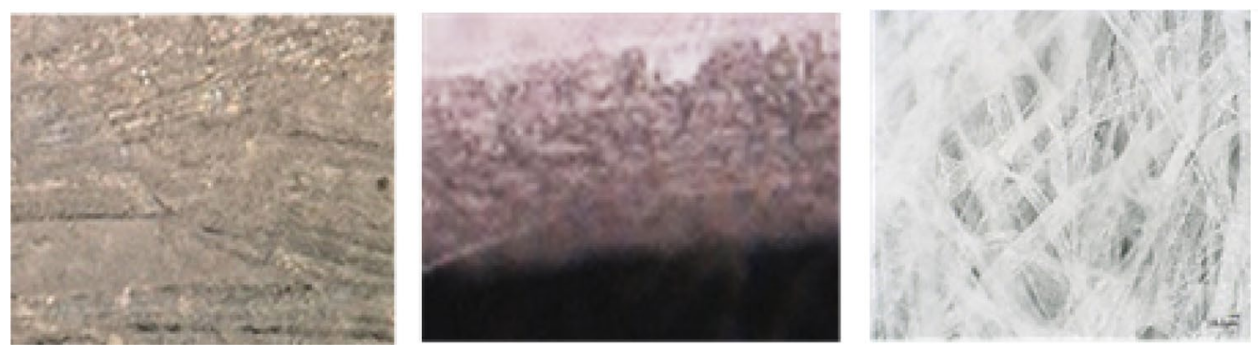

SN Applied Sciences A SPRINGer Nature journal 


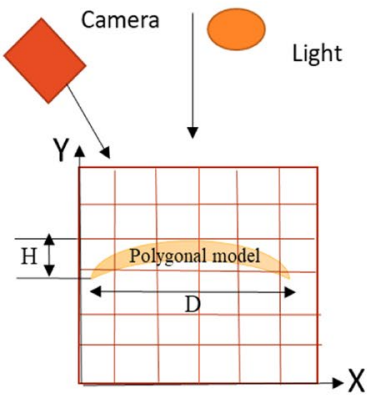

(a)

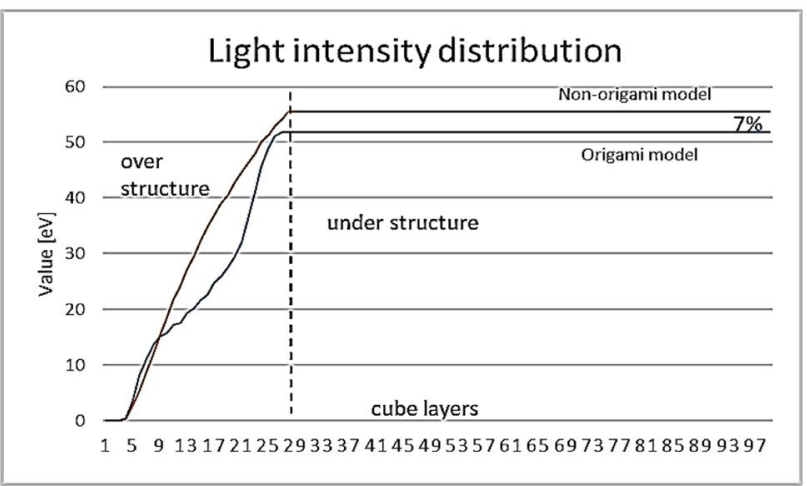

(b)
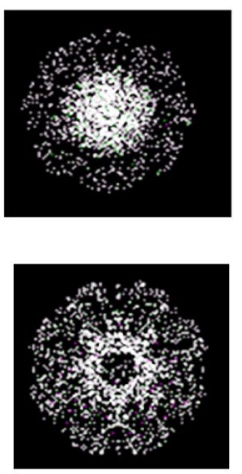

(c)
Fig. 4 The ray tracing simulation: a the simulation scheme, b graphs of the resulting data in the layers of the cube, where the total photon energy is shown on the vertical axis. The area occu-

tracing rays of light with wavelengths of $400 \mathrm{~nm}, 300 \mathrm{~nm}$ and $250 \mathrm{~nm}$ with correspondent refraction indices are used to calculate the integral value of the photon energy $E$ (in eV) for each sub-cube. In the RRT algorithm, we consider light intensity $L I$ as the rate at which light energy is delivered to a sub-cube. It is calculated as follows:

$L I=k_{\mathrm{rt}} \cdot E$

where $k_{\mathrm{rt}}$ is a coefficient that determines the decrease in light intensity due to reflection and transmission during light propagation through the $n$ layers of the structures under study. The values of this coefficient are calculated by using the Eqs. 6, 7 (the initial value is 1.0). Each ray is continued until it ended up as reflection or transmission from the cube. To reduce the time and efforts required in ray tracing some of the rays are discontinued after 7 levels of recursion. Total light intensity $L L I$, which we call light intensity in the cube layer, is determined as follows:

$L L I=\sum_{1}^{n} \sum_{1}^{m} L_{m}$

where $n$ is the number of sub-cubes in the cube layer, $m$ is the number of rays in the sub-cube.

The light rays enter into the cube in the direction perpendicular to its upper facet and fall on the surface of the embedded model along the $Y$-axis (Fig. 4a). In each run, the $128 \times 128$ light rays are used. The bottom layer of the cube is considering as a ground where usually humans are located under a beach umbrella. Since paper umbrellas are often used as sun protection, in this study, we consider paper with a thickness of $0.1 \mathrm{~mm}$ as a possible material for origami-based model. The optical paper parameters: basic weight $\mathrm{W}=70 \mathrm{~g} / \mathrm{m}^{2}, \mathrm{~S}=49 \mathrm{~m}^{2} / \mathrm{kg}, \mathrm{K}=5.5 \mathrm{~m}^{2} / \mathrm{kg} . R_{0}=0.6$ and $T=0.2$ are calculated as discussed in Sect. 4.1. It should pied by the model in the cube (cube layers from 4 to 28 ) is called the "over structure", c the images of light intensity distribution: the non-origami model (top) and the origami model (bottom)

be noted, that using derivatives of refractive index $n$, i.e. the group of indexes with small deviations from the main refractive index $n=1.56$, practically, gives almost the same results of calculating light intensity as with the fixed refraction index. The RRT simulation of the propagation of rays through the studied structures shows a decrease in light intensity by $7 \%$ in the area under the origami structure compared to the non-origami structure (Fig. 4b). Figure 4c shows the distribution of light intensity at the bottom of the cube for the non-origami and origami structures (data are shown in white). Based on the visualization results, it is possible to conclude that there is a higher concentration of rays in the area under the non-origami structure in comparison with the origami structure in the same area. Taking into account the results obtained with regard to UV light, it can be concluded that the harmful effect on human health can also be reduced with the use of the origami-based umbrella.

\subsection{Shape optimization of the origami-based umbrella}

According to simulation results, we consider an optimal design of the given geometry model that is optimal with respect to the basic designed origami model. The criterion for the distribution of light intensity using geometric field tracing is implemented in the form of the RRT algorithm. An elegant example of using evolutionary optimization technique to create a curved refractive interface in the eye of a certain type of trilobite (phacopida) is presented in [35]. Examples of applying an evolutionary optimization technique for designing a shape under light constrains has been discussed in several papers, for instance [36]. Driven by the successful modelling of phacopida eye, we tried to continue 
consideration of the applicability of the combination of ray tracing and the optimization techniques based on Genetic Algorithms (GA) for the modification of the model shape. The most attractive feature of the GAs is that they are easy to interface with a model. The main drawback of GAs is that the optimization problem can be trapped in a local minimum. We apply the $\mathrm{C}++$ genetic algorithm objects library GAlib [37]. For the optimal design of the given model, coordinates of vertex $V_{i}$ of the 3D polygonal origami model (optimization parameters), where each $V_{i} \in E^{3}$ has the corresponding valency equal to 4 (Fig. 5a), are modified according to a scheme shown in Fig. 5b. Our goal is to optimize the geometric parameters of this model, while maintaining the folding properties and preserve the flatness of the neighboring quadrilateral elements of the model. For that, the coordinates of meridian points belonging to the quadrilateral element of the plane passing through the circle center $o$, vertex $V_{i}$, and pole of the sphere $p$, as shown in Fig. $5 \mathrm{~b}$, are also modified to avoid skewing. The resulting warped shape is evaluated with the help of the RRT algorithm, which allows us to take into account a collection of light intensities of refracted and reflected rays stored in the area under the origami-based umbrella structure. Changing the coordinates of the nodes naturally leads to a variation of the coordinates of the nodes belonging to the vertex ring of the basic model. As the result, the initial shape of the model is modified (Fig. 5c).
The new umbrella structures (Fig. 5c) with higher fitness join the population to replace those previous ones whose fitness measures are lower. We calculate a fitness function that expresses a fact that the lower intensity we get in the area under the umbrella the more effective shading can be achieved. Our experiments show that with the evolutionary optimization of the geometry of the model, we can improve the shading effect with respect to the basic design (Fig. $5 \mathrm{~d}$ ) by about 3\% (Fig. 5e). The experiments also showed that we have no problems with the convergence of the algorithm. As it was noticed in [35], the problem of local trapping usually arises when the optimization process is forced to make a decision between different ways of increasing fitness, for example, if many variables in the system are allowed to be free. In our experiments, the optimization procedure is able to converge to the optimal solution even if we increase the number of parameters such as the optical material properties (the results are not shown). From our point of view, the convergence problem may arise in view of the "badly" defined scheme of selecting the points that define shape deformation. However, in the presented research study, the modification scheme discussed above allows for attaining reduction of light intensity accumulated in the area under the umbrella by $10 \%$ with respect to the initial "bad" design. Nevertheless, finding the best scheme of selecting points that defines shape deformation is an open question. Fortunately, as mentioned in [38], an algorithm can have

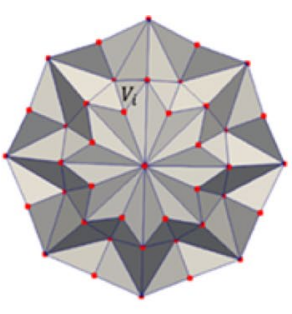

(a)

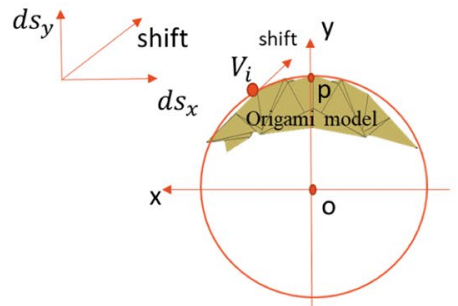

(b)

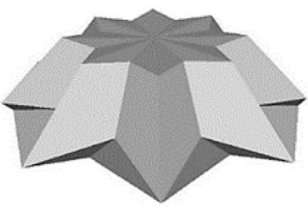

(c)

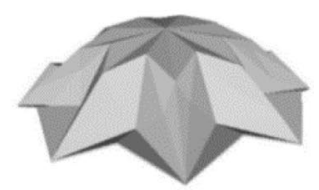

(d)

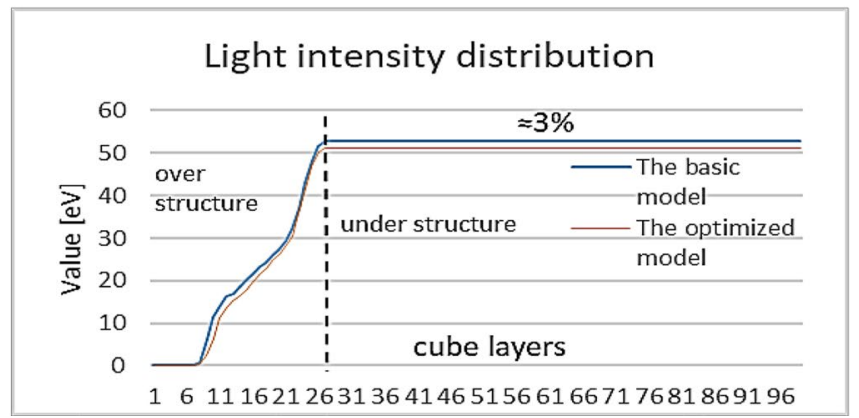

(e)

Fig. 5 The optimization technique: a the model vertices (red dots), b the 2D scheme of the model modification, where shift is a vector defining possible variation of the coordinates of vertex $V i$ in the meridian plane; $d s_{x}$ and $d s_{y}$ are displacements of the coordinates, $c$ the 3D optimal model, $\mathbf{d}$ the 3D basic model, e graphs of simulation results, where the total photon energy shown on the vertical axis and the area between the layers 6 and 26 is the "over structure" 
practical significance even if it fails to identify the true global optimal solution because the algorithm can find alternative solutions that more fully satisfy some minimal set of user-defined requirements.

\section{FE simulation of folding the model and structure behavior under lateral loading}

In the last years, the structural analysis of the folded shell designs and their mechanical properties have been discussed in many papers, for instance $[39,40]$, where the aim is to describe the kinematic and structural properties, to present and find suitable modelling methods to capture structural salient behavior. A non-linear formulation for performing static/quasi-static analyses of origami structures based on 'bar-and-hinge' models is proposed in [41]. In [42], FE models of the origami-based shelter structures are the subjects of the numerical simulation of the structure behavior under gravitational and lateral nodal forces using eigenvalue buckling analysis and non-linear static analysis with SAP2000 program. The wind-load can be very destructive because it generates pressure against the surface of a structure. Wind-load on the surface is considered as the lateral nodal force. The origami-based umbrella model should be studied using numerical simulations of falling or breaking in windy conditions. We assume that the developed 3D model must be a rigid structure, because many stiffeners are created by the origami design that provides a structural stiffness. The given model shape deformation can be demonstrated by the FEM simulation results. Unlike light propagation modelling, in this simulation we must use more durable material than paper such as lightweight synthetic polypropylene (PP), a plasticcoated paper, or cardboard (CB), which are modelled as linear elastic materials. The stabilizing systems in PP fibers give a degree of UV resistance and absorb any moisture, so that their dimensions do not vary with changing humidity or when they become wet. PP is a mechanically rugged material that is used in storage, packaging, other suitable applications. Cardboard is a paper-based product having a greater thickness and mechanical properties that are suitable for origami-based structures. Modelling and kinematic analysis of cardboard folding products is a research interest particularly for the packaging industry [43].

\subsection{Displacement analysis of the model deformation}

Numerical simulations are carried out using the commercial FEA software ANSYS R19.2 to show the structure behavior under the applied loads. The FE origami and nonorigami models are given by the geometrical parameters: the diameter $\mathrm{D}$ and the height $\mathrm{H}$ (Fig. 6a). These structures are modelled using the shell triangular elements. The forces $\mathrm{F}$ in the range from $2 \mathrm{~N}$ to $30 \mathrm{~N}$ are applied in the Z-axis direction (the global $X, Y, Z$ coordinate system) to the nodes of the half-parts of the shell models. As shown in Fig. 6b, c, the force is distributed over the point-loads according to the colour arrows (red arrows: max value force $F$, blue arrows: $F / 2$, yellow arrows: $F / 4$ ) by analogy with the scheme of the lateral force loading proposed in [42]. Another half-part of the shell model, including the central parts, is considered as the fixed part to restrict lateral movement (the red squares and a circle). The static structural analysis of the model deformation is employed to define the localized stresses in the model under lateral loading. To make the lightweight design, the thickness of the material (PP and $\mathrm{CB}$ ) is taken $1 \mathrm{~mm}$. Based on the preliminary simulation results, $C B$ is not acceptable as a material for the non-origami structure with a thickness less than $2 \mathrm{~mm}$ due to structural failure when force value is $20 \mathrm{~N}$. Graphs of Von-Mises stress versus the nodal displacement in the $Z$-axis (the maximum value is given from the range of displacement) display simulation results for the origami and non-origami models in the given force range from 0 to $20 \mathrm{~N}$ (Fig. 7a, c). Figure 7b, $d$ show stress distribution over the FE origami model and non-origami models. Origami-based umbrella structure exhibits approximately linear response over a small range of load intensities and the shape of the origami model

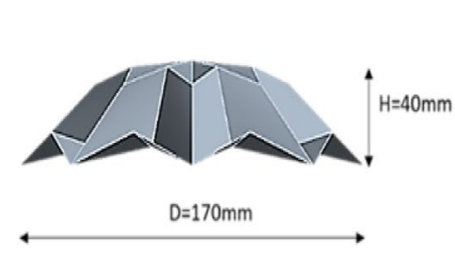

(a)

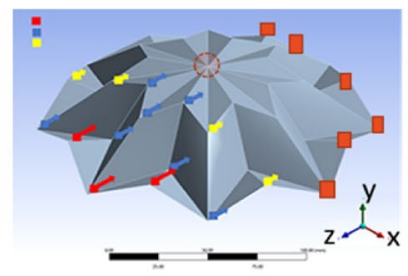

(b)

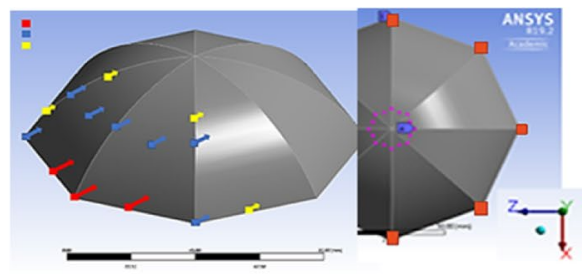

(c)

Fig. 6 The FE models: a the model geometry, $\mathbf{b}$ the scheme of the force distribution and the fixed parts for the origami model, $\mathbf{c}$ the scheme of the force distribution and the fixed parts for the non-origami model with the same geometrical parameters as the origami-based model 


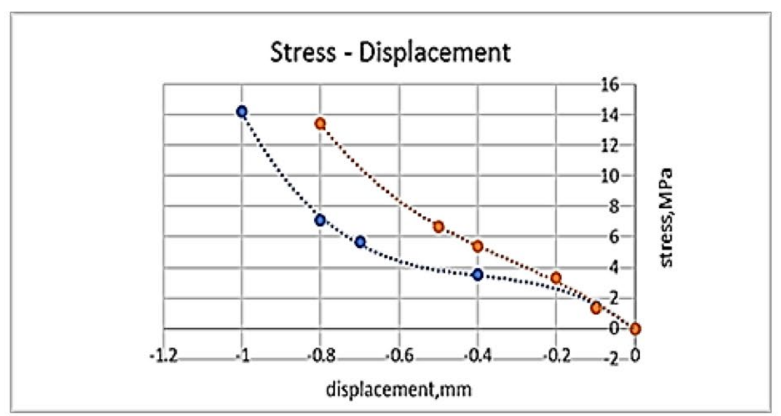

(a)

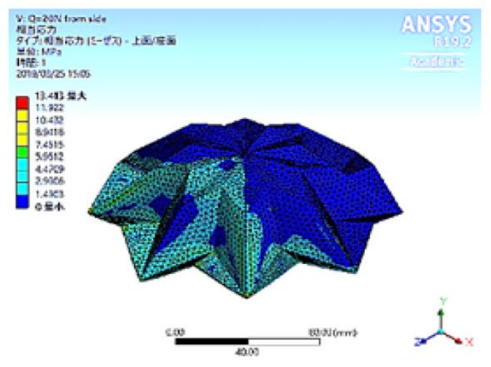

(b)

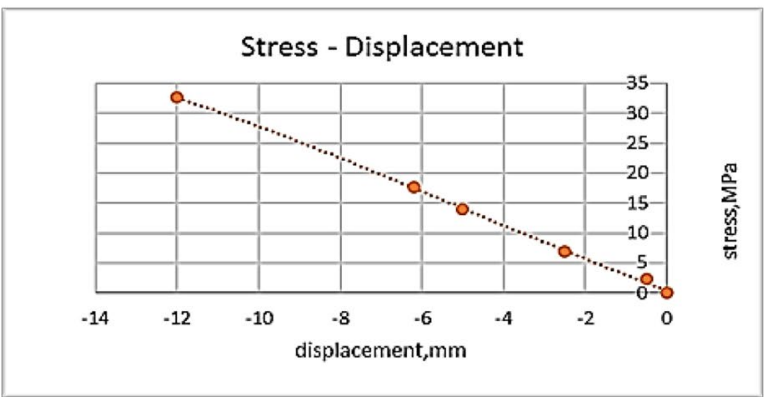

(c)

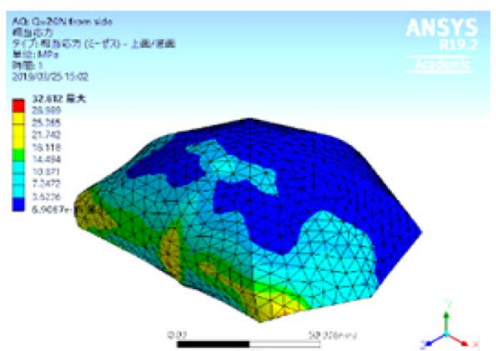

(d)

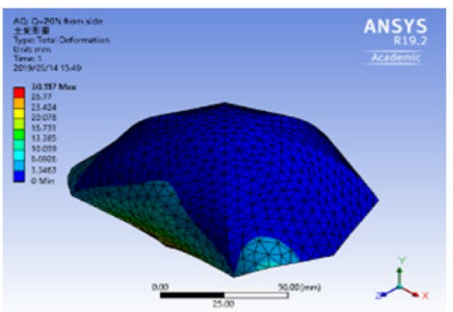

(f)
Fig. 7 FEA results: a graphs of simulation results (the origamimodel, material CB is marked as blue dots; PP material is marked as orange dots), b stress distribution over the origami model nodes $(F=20 \mathrm{~N}, \mathrm{PP}$ material), c graph of simulation results (the non-origami model, PP material), $\mathbf{d}$ stress distribution over the

is slightly deformed compared to the large deformation of the non-origami model. Under higher forces, the stiffness of the origami-based structure is leading to a nonlinear response that allows the structure to dissipate the energy of the wind-loads. For the origami-based model, the maximum stress value is equal to $14 \mathrm{MPa}(\mathrm{F}=20 \mathrm{~N})$ in comparison with yield stress $\sigma_{y}=30 \mathrm{MPa}$ for PP material. With the same material properties, the non-origami (the traditional umbrella) model demonstrates linear response under loads and the maximum stress value that is equal to $32 \mathrm{MPa}(\mathrm{F}=20 \mathrm{~N})$. It means that an origami-based structure can provide a double factor of safety in comparison with the model of the traditional umbrella with the same material. The maximum value of the total deformation (PP material), which is the root mean square (RMS) value of directional (X, Y, Z-coordinates) deformations, obtains the maximal nodal displacements under stresses in the intervals from $22.36 \mathrm{~mm}$ to $25.16 \mathrm{~mm}$ for the origami model non-origami model nodes ( $F=20 \mathrm{~N}, \mathrm{PP}$ material), e the total deformation distribution over the origami model ( $F=20 \mathrm{~N}$, PP material), $f$ the total deformation distribution over the non-origami model $(F=20 \mathrm{~N}, \mathrm{PP}$ material)

and from 26.77 to $30.12 \mathrm{~mm}$ for the non-origami model (Fig. 7e, f). As can be seen, the origami-based model shows greater resistance to loads using PP material ( $1 \mathrm{~mm}$ thick) compared to the non-origami model when using the same material with the same thickness.

\subsection{Kinematic modelling and dynamic analysis of the behavior of the origami-based structure}

The kinematic model of the origami-based umbrella as a simplified model provides insight into the essential global behavior of the considered structure where the hinges are located in the mountain and valley crease lines according to the Axis-Shift method [44, 45]. Figure 8 a shows the kinematic scheme of the unit-cell that is modelled as a set of thick panels. To avoid crease intersections, the intersection points are cut out in the simulation model. Solid mesh is generated from $2.5 \mathrm{~mm}$ surface triangle mesh. 


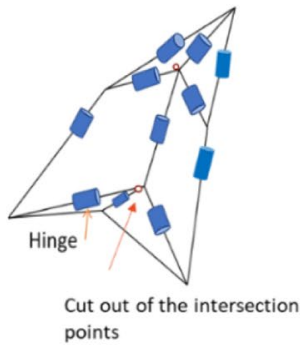

(a)

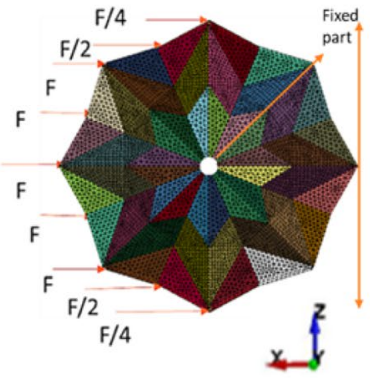

(d)

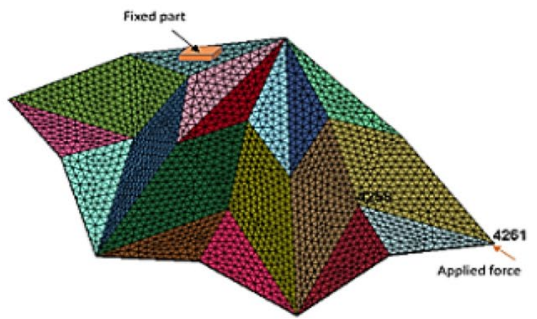

(b)

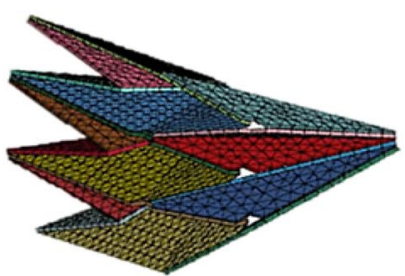

(c)

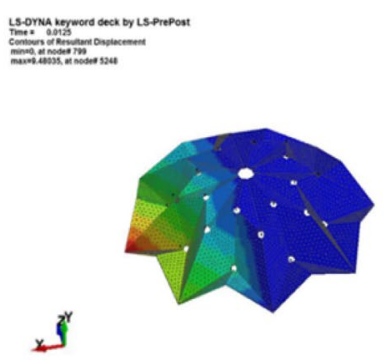

(e)

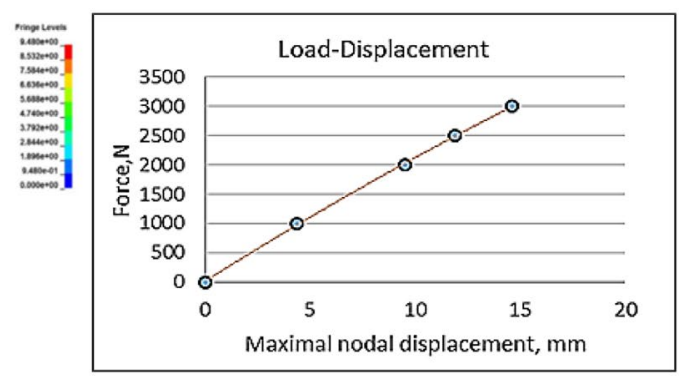

(f)
Fig. 8 The FE simulation: a the kinematic scheme for the unit-cell, b the FE simulation model (the 3 unit-cells), c simulation results of the folding process, $\mathbf{d}$ the scheme of the force applications, e the

Here, we demonstrate the results of the FE simulations for two cases: (1) the folding process for the part (the 3 unit-cells) of the model; (2) the nodal displacement under lateral loading for the full model. Numerical simulations of the structural behaviors of the given model (with the same geometrical parameters, as shown in Fig. 6a) are done using LS-DYNA commercial software for both cases. In the first case, the material is a plastic-coated paper (type of cardboard) with a thickness of $1 \mathrm{~mm}$. One thick panel of the model is considered as a fixed part and the force $F$ of $500 \mathrm{~N}$ is applied to boundary nodes along the radius of the model (Fig. 8b). The simulation result demonstrates the compact packaging of the origami-based umbrella (Fig. 8c). In the case of lateral loading, the nodal force $F$ is distributed over the point-loads in the $X$-axis direction (the global $X, Y, Z$ coordinate system) as shown in Fig. $8 \mathrm{~d}$. The force loading scheme differs from the static analysis discussed above: for static analysis, we need to use a more complex loading scheme than with the kinematic approach. In kinematic modelling, the lateral load distributed over a smaller number of point-loads (Fig. 8d) provides the motion of the joints, which connect the rigid faces of the unit-cells (kinematic chain) in accordance with their sequence determined by the pattern of the 3D model. PP is used as the material with a thickness of $1 \mathrm{~mm}$. The structure behavior under the lateral force $F=2000 \mathrm{~N}$ is illustrated in Fig. 8e. The deformed part of the model shows folding motion according to the kinematic scheme. maximal deformation over the model, $\mathbf{f}$ the line graph of the result maximal nodal displacement

The graph in Fig. $8 \mathrm{f}$ demonstrates the correlation between lateral force $F$ in the range from 1000 to $3000 \mathrm{~N}$ and the resultant maximal nodal displacements. Increasing in nodal force leads to increasing in nodal displacement: $\mathrm{F}=1000 \mathrm{~N}$ gives the maximum displacement of $4.4 \mathrm{~mm}$. For $\mathrm{F}=3000 \mathrm{~N}$ the maximum displacement is $14.6 \mathrm{~mm}$. That force value is the critical load value because of the slight destruction of the structure. The origami structure does not go back to its initial state after releasing the applied loads and stays in a deformed state because of existing sharp creases [46] but the shape can be improved by applying human force.

\section{Discussion}

In the current research, the basic designed origamibased model of the beach umbrella presented in Sect. 3 is used to examine its reflection/refraction properties and mechanical properties under lateral forces as well as folding behavior. The combination of materials, which we use in the simulations (shown above), can be realized in the design of the umbrella, which can be offered: the lightweight origami-based framed structure for the sun umbrella (Fig. 9a-d) to meet the reflective and mechanical properties of the origami-based structure that are shown above. The frame is a rigid foldable structure based on the same crease pattern shown in Fig. 2a. Facets are cut 


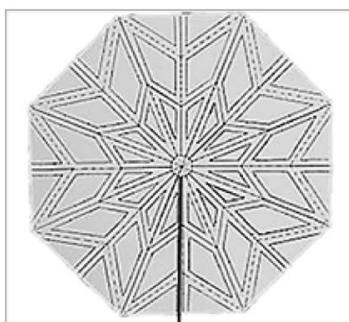

(a)

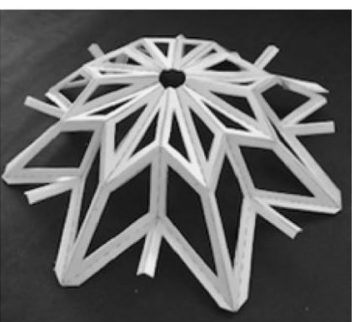

(b)

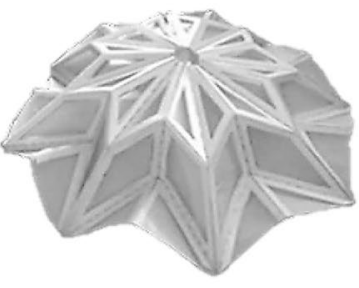

(c)

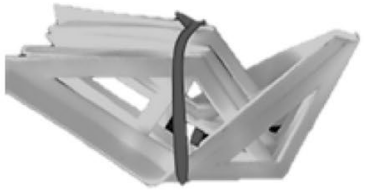

(d)

Fig. 9 The framed design: $\mathbf{a}$ the 2D crease pattern (the cutting line is marked as the black line), $\mathbf{b}$ the plastic-cardboard frame, $\mathbf{c}$ the framed model with washi-paper covering, $\mathbf{d}$ compact packaging

out and remove. Voids formed are covered with a suitable material such as paper, washi-paper (the thickness of materials is in the range of $0.13-0.18 \mathrm{~mm}$ ), or thin plastic film. Washi-paper can be useful because of low weight, the strength, the water-resistance, and the good UV protection [47]. The frame can be made from the material sheets (1 mm thick) such as PP, plastic-cardboard, or plywood. This type of structure satisfies the mathematics rules for producing flat-foldable origami and shows the compact final shape.

\section{Conclusions}

This article demonstrates the optical properties of the developed origami-based structure and considers the possibility of using origami-based beach umbrellas to reduce the effects of sunlight on human health. The structure behavior under the applied loads also is the subject of the research. FE simulation models have been developed to study the effects of a structured model, such as the origami-based model and the non-origami model, on light propagation efficiency and lateral load resistance.

In this study, we show that origami-based structures (umbrellas or shields) with using paper as a material can provide reasonable decreasing light intensity in the area under the structures. Ray tracing results show that light intensity can be decreased by $7-30 \%$ (varies with modelling conditions) in comparison with the nonorigami structure (the traditional umbrella type), which is important for human health. UV coating and lamination of paper have advantages for decreasing the harmful effect on human health; however, such technology increases the cost of paper. The studied paper origamibased model demonstrates promising performance as a suitable screen for sunlight protection. The evolutionary optimization of the geometry of the model shows that the shading effect with respect to the basic design can be improved by about $3 \%$. We believe that the value of light intensity can be used as a general measure of assessment for any origami structures in the entire spectrum of electromagnetic radiation.

The FE simulation approach is employed to study the mechanical behavior of the presented origami-based structure using polypropylene and cardboard as materials. Simulation results demonstrate that the origami-based umbrella structure provides good wind-load resistance and it is compact when stowed.

As a material for the basic umbrella model and for the framed design, cardboard, plastic- cardboard, or plywood can be suggested. Plastic films, fabric, laminated paper, washi-paper, and an ordinary paper are suitable as the covering for the framed structure. For the lightweight framed structures, the multi-thickness materials also can be used.

Indoor experiments with the scaled basic models demonstrate decreasing irradiance power in 14-30\% under the origami-based model using washi and an ordinary paper in comparison with the non-origami model with the same materials and conditions of experiments. UV irradiance power (UV wavelength: from 260 to $395 \mathrm{~nm}$ ) was measured by UV light meter CENTER 532 (measurement range: $1-3999 \mathrm{uw} / \mathrm{cm}^{2}$ ). As a source of UV light, 9UV-LED light (PW-UV043H-04, the maximum wavelength equal to $375 \mathrm{~nm})$ is used.

The results of the research presented in this article are of interest to the potential use of the origami-based structures related to the protection from the harmful effects of UV radiation on human health. Replacing mechanical parts, such as strings and hinges in the traditional umbrella, by the origami-based structure can be considered as the alternative way of the beach umbrella design.

To confirm the results obtained in this study, more researches and experiments would need to be conducted. Outdoor experiments with the real size structures for testing optimal shapes and materials for increasing the shielding effect as well as manufacturing the non-paper umbrella structures with using laser cutting, 3D printing, or robotics are considered as future works. 
In the future, one of the directions of using the RRT algorithm might be the development of models of reflection/refraction/absorption of light in various types of paper structures based on the analysis of their microscopic images. For further study, physical optics for considering the wave properties of light should be taken into account for deeper investigation of the UV protective properties of the origami-based structure. We might consider expanding the application of ray tracing modelling to origamiinspired structures for other ranges of electromagnetic frequencies. These structures should be used with respect to electromagnetic phenomena in engineering applications.

Acknowledgements We acknowledge Meiji Institute for Advanced Study of Mathematical Sciences, Meiji University (Tokyo, Japan) for financial support of this research (MIMS Joint Research Project 2018). We would like to thank Professor A. B. Samokhin, (Russian Technological University - MIREA, Moscow, Russia) for valuable guidance and advice. We would like to take this opportunity to thank Professor N.J. Suematsu (Meiji University, Tokyo, Japan) for preparing microscopic images of paper structures for our research.

Author's contribution MS designed the origami-base umbrella, performed the experiments and the analysis of the numerical simulation results. VS developed ray tracing algorithm and produced GA optimization. AA, PTT and IH performed the numerical simulations and the analysis of the FE simulation results. All the authors participated in manuscript writing.

\section{Compliance with ethical standards}

Conflict of interest The authors declare that they have no conflict of interests.

\section{References}

1. World Health Organization. https://www.who.int/uv/sun_prote ction/en/

2. Yu Y (2015) UV interactions with fibres and fibrous structures. Dissertation, Deakin University, Australia. http://hdl.handl e.net/10536/DRO/DU:30084236

3. Buchwald JZ (1989) The rise of the wave theory of light: optical theory and experiment in the early nineteenth century. University of Chicago Press, Chicago. ISBN 0-226-07886-8

4. Lvovsky Al (2013) Fresnel equations. In: Driggers RG (ed) Encyclopedia of optical engineering. Taylor and Francis, New York, pp 1-6. https://doi.org/10.1081/E-EOE-120047133

5. Fresnel Equations (1970-1979) The great Soviet encyclopedia, 3rd edn. The Gale Group Inc. https://encyclopedia2.thefreedic tionary.com/ Fresnel + Equations

6. Mrna L, Rihacek J, Sarbort M, Hornik P (2019) Solar absorber with a structured surface-a way to increase efficiency. Acta Polytech 59(02):134-143

7. Catalkaya I, Kent S (2012) Analysis of multiple wedges electromagnetic wave absorbers. J Prog Electromagn Res M 26:1-9

8. Turnel N, Goodwine B, Sen B (2015) A review of origami and its application in mechanical engineering. In: ARCHIVE proceedings of the institution of mechanical engineers part $\mathrm{C}$ journal of mechanical engineering science 1989-1996 (vols 203-210) 230(14). https://doi.org/10.1177/0954406215597713
9. Myer JN, Cooke F (1969) Optigami-a tool for optical system design. Appl Opt 8(2):260-269

10. Pehrson NA, Magleby SP, Lang RJ, Howell LL (2016) Introduction of monolithic origami with thick-sheet materials. In: Proceedings of the IASS annual symposium 2016 "Spatial Structures in the 21st Century". https://www.researchgate.net/publicatio $\mathrm{n} / 311510862$

11. Lv C, Krishnaraju D, Konjevod G, Yu H, Jiang H (2014) Origami based mechanical metamaterials. Sci Rep 4:5979-5981

12. Kuribayashi $K$, Tsuchiya $K$, Zhong $Y$, Tomus $D$, Umemoto $M$, Ito T, Sasaki M (2006) Self-deployable origami stent grafts as a biomedical application of Ni-rich TiNi shape memory alloy foil. J Mater Sci Eng A 419:131-137

13. Miyashita S, Guitron S, Li S, Rus D (2017) Robotic metamorphosis by origami exoskeletons. J Sci Robot 2(10):eaao4369. https://doi. org/10.1126/scirobotics.aao4369

14. Sessions D, Fuchi K, Pallampati S, Grayson D, Seiler S, Bazzan G, Reich G, Buskohl Ph, Huff G (2018) Investigation of folddepended behavior in an origami-inspired FSS under normal incidence. J Prog Electromagn Res M 63:131-139

15. Fuchi K, Buskohl PR, Joo JJ, Reich GW (2016) Control of RF transmission characteristics through origami design. In: Proceedings of the ASME 2016 international design engineering technical conferences and computers and information in engineering conference, paper no. DETC2016-59899, 05BT07A018. https:// doi.org/10.1115/detc2016-59899

16. Fuchi K, Buskohl PR, Joo JJ, Reich GW, Vaia RA (2016) Numerical analysis of origami structures through modified frame elements. In: Origami ${ }^{6}$ : Proceedings of the sixth international meeting of origami science. Mathematics, and Education, pp 385-395

17. Chen Y, Feng J (2018) Kinematic indeterminacy and folding behavior of a class of overconstrained frameworks with symmetry. J Acta Mech 229(3):1157-1169. https://doi.org/10.1007/ s00707-017-2044-8

18. Grifoni D, Carreras G, Sabatini F, Zipoli G (2005) UV hazard on a summer's day under Mediterranean conditions, and the protective role of a beach umbrella. Int J Biometeorol 50(2):75-82

19. Vejakupta $K$, Udompataikul $M$ (2014) Umbrella with ultraviolet radiation protection. J Cosmet Dermatol Sci Appl 4:228-233. https://doi.org/10.4236/jcdsa.2014.4403

20. Maity S, Singha KP, Debnath P, Singha M (2013) Textiles in electromagnetic radiation protection. J Saf Eng 2(2):11-19

21. Turnbull DJ, Parisi AV (2005) Increasing the ultraviolet protection provided by shade structures. J Photochem Photobiol B Biol 78:61-67

22. Moise AF, Aynesley R (1999) Ambient ultraviolet radiation levels in public shade settings. Int J Biomet 43:128-138

23. Miura K (1980) Method of packaging and deployment of large membranes in space. In: Proceedings of the 31 st congress international astronautical federation, $\mathrm{pp}$ 1-10

24. Kawasaki T (1989) Proceedings of the first international meeting of origami science and technology (Huzita, H.), pp 229-237

25. Zhao Y, Kanamori Y, Mitani J (2016) Geometry of axisymmetric 3D origami consisting of triangle facets. In: Proceedings of 17th international conf. on geometry and graphics, paper $\mathrm{N} 11$. https ://www.researchgate.net/publication/316465041

26. Born M, Wolf E (1989) Principles of optics. In: Sixth corrected ed. Pergamon, Oxford

27. Elvins TT (1992) A survey of algorithms for volume visualization. Comput Graph 26(3):194-201

28. Kubelka P (1948) New contributions to the optics of intensely light-scattering materials. Part 1: Nonhomogeneous layers. J Opt Soc Am 38(5):448-457

29. Kubelka P (1954) New contributions to the optics of intensely light-scattering materials. Part II: Nonhomogeneous layers. J Opt Soc Am 44(4):330-334 
30. Oren M, Nayar SK (1994) Generalization of Lambert's reflectance model. In: Proceedings of SIGGRAPH, pp 239-246

31. Carlsson J, Persson W, Hellentin P, Malmqvist L (1995) The propagation of light in paper: modelling and Monte- Carlo simulations. In: Proceedings of the international paper physics conference, pp 83-86

32. Bjuggren $M$, Quinteros $T$, Béland $M-C$, Krummenacher L, Mattsson L (1997) Light and paper: progress report 1995-1996. In: Institute of optical research technical report, vol 316

33. Hainzl R, Berglind R, Bjuggren M, Beland MC, Quinteros T, Granberg $\mathrm{H}$, Mattsson L (2000) A new light scattering model for simulating the interaction between light and paper. In: Proceedings of the TAPPI international printing and graphic arts conference, pp 9-17

34. Raunio JP (2014) Quality Characterization of tissue and newsprint paper based on image measurement. Dissertation, Tampere University of Technology, Publication 1270. ISBN 978-95215-3416-4, ISSN 1459-2045

35. Goel M, Thompson RL (1988) The evolution of the trilobite eye. In: Computer simulations of self-organization in biological systems, part 11. Croom Helm, London, pp 275-290

36. Savchenko VV, Pasko AA. (1999) Evolutionary Optimization of Functionally Defined Shapes: Case study of natural optical objects. In: Proceeding of the computer graphics international conference, pp 20-24

37. GAlib. http://lancet.mit.edu/ga/

38. Kuper T (1992) Global optimization finds alternative lens design. J Laser Focus World 28(5):193-195

39. Schenk M (2011) Folded shell structures. Dissertation, University of Cambridge

40. Schenk M, Guest SD (2011) Origami folding: a structural engineering approach. In: Origami 5. CRC Press, Boca Raton, pp 293-305

41. Liu K, Paulino GH (2017) Nonlinear mechanics of non-rigid origami: an efficient computational approach. Proc R Soc A 473:20170348. https://doi.org/10.1098/rspa.2017.0348
42. Norman M, Arjomandi K (2017) Origami applications in structural engineering: a look at temporary shelters. In: Proceedings of the 2-nd world congress on civil, structural, and environmental engineering (CSEE'17), paper no ICSENM 130. ISSN: 2371-52 94. https://doi.org/10.11159/icsenm17.130

43. Qiu C, Amizadeh V, Dai JS (2013) Kinematic and stiffness analysis of an origami-type carton. In: Proceedings of the ASME 2013 international design engineering technical conferences and computers and information in engineering conference IDETC/ CIE DETC2013-12343 (DRAFT)

44. Tachi T (2011) Rigid-foldable thick origami. In: Origami 5: Proceedings of the fifth international meeting of origami science, mathematics and education, pp 253-263

45. Thai PT, Savchenko M, Hagiwara I (2018) Finite element simulation of robotic origami folding. J Simul Model Pract Theory $84: 251-267$

46. Lv C (2016) Theoretical and finite element analysis of origami and kirigami based structures. Dissertation, Arizona State University. https://repository.asu.edu/attachments/174993/conte nt/Lv_asu_0010E_16244.pdf

47. Ohsawa I, Takahashi J, Uzawa K, Kanai M, Murayama H, Kageyama K (2007) Tensile properties of washi-paper reinforced polylactic acid (PLA) as a green composite. In: Proceedings of the 10th Japan international SAMPE symposium and exhibition (JISSE-10), (Green Composites-2-2)-1-6

Publisher's Note Springer Nature remains neutral with regard to jurisdictional claims in published maps and institutional affiliations. 